\title{
Implicit Preconceptions of Male and Female Intelligence in Contemporary Russia
}

\author{
Victoria A. Dessau, Irina A. Mironenko* \\ Department of Psychology and Communication Disorders, State Pushkin University, St. Petersburg, 196605, Russia
}

\begin{abstract}
Investigation revealed implicit preconceptions of Male and Female Intelligence in contemporary Russian society. A substantial difference in the structure and constituting elements between the representations was shown. It was shown also that preconceptions of Male and Female Intelligence are substantially different for male and for female samples. A two-stage procedure was used. At the first stage our subjects freely described Male and Female intelligence and at the second stage we used two sets of rating scales. One of them was a set for personality traits ("Personality Semantic Differential") and another one was specially constructed of nine scales for evaluating cognitive abilities.
\end{abstract}

Keywords Implicit Theories, Lay Theories of Intelligence, Wisdom, Gender Stereotypes, Gender Differences in Intelligence Estimates, Male and Female Intelligence

\section{Introduction}

Few topics in psychology are as popular as gender differences in intelligence and related issues are seldom out of attention both of academics and broad public.

There is a general "academic consensus for many years to the effect that actual differences between the sexes are minimal" [1, p. 513]. The dominating view is that "the sex difference in psychometric $\mathrm{g}$ is either totally non-existent or is of uncertain direction and inconsequential magnitude" [2, p. 540].

Nevertheless, studies on lay theories of intelligence show consistent differences[3-7]. Moreover, there is a lot of evidence that at the level of everyday perception intellectuality is a masculine characteristic[4-9]. Also a lot of data show that these differences are culture biased[1,6,10-13].

Our research was aimed to investigate gender differences in lay theories of intelligence in contemporary Russian society. The investigation was designed in order to explore implicit preconceptions of Male and Female Intelligence.

We used a two-stage procedure, so that the results of the first stage experiment were employed for the design of the second stage. At the first stage our subjects were asked to write down as many characteristics of Male Intelligence and then of Female Intelligence as possible. So they described Male and Female Intelligence freely. At the second stage rating scales and semantic differential technique were used.

358 subjects were involved. All of them were residents of

* Corresponding author:

mironenko_i@mail.ru (Irina A. Mironenko)

Published online at http://journal.sapub.org/ijpbs

Copyright (C) 2011 Scientific \& Academic Publishing. All Rights Reserved
St.Petersburg(Russia), adults, aged 20 to 45, including 105 men( 26 years old on average) and 253 women( 29 years old on average).

\section{Experiment 1}

At the first stage our subjects freely described Male and Female Intelligence.

\subsection{Method}

At this stage of investigation respondents were asked to write down as many features of Male and then Female Intelligence as possible ("free responses").

The resulting data contained nearly 1500 words which reflect qualities of Male and Female Intelligence as perceived by our respondents. Afterwards the data were subjected to content analysis and further on to cluster analysis.

\subsection{Data Analysis}

Coding process was used which ended in grouping of words with similar meaning into descriptive categories, which resulted in 32 categories. Then each of them was given a title and a definition.

Once the categories were identified, the frequency of each category was crossed with respondents' belonging to the male or female part of the sample.

At this stage we considered inverse categories separately (Friendliness and Hostility; Extraversion and Unsociability; etc.).

\subsection{Results and Discussion}

It should be noted that among the resulting categories we 
have found not only those applicable to cognitive processes, but also the qualities which are usually referred to as personality traits: Decisiveness, will power; Refinement; Extraversion; Sense of humor; etc. Among the characteristics ascribed to intelligence we have detected not only those which are usually regarded as positive features but negative as well, usually attributed to the opposite sex: Hostility, Stupidity, Recklessness.
The results revealed existence of some characteristics which were mentioned by the majority of our respondents while describing both Male and Female Intelligence: Extraversion; Friendliness; Decisiveness, will power; Emotional stability; Refinement and culture; Ability to understand and predict others; Logic, common sense; Using knowledge in practice; Knowledge of life, as shown in Table 1 (results given in \% from the total number of respondents).

Table 1. Number of Respondents Naming the Categories Describing Male or Female Intelligence.

\begin{tabular}{|c|c|c|c|c|}
\hline \multirow{2}{*}{ Categories } & \multicolumn{2}{|c|}{ Male Intelligence } & \multicolumn{2}{|c|}{ Female Intelligence } \\
\hline & Male Sample & Female Sample & Male Sample & Female Sample \\
\hline Extraversion & 38,0 & 37,8 & 48,0 & $35,6^{*}$ \\
\hline Unsociability & 6,0 & 7,8 & 8,0 & 7,8 \\
\hline Friendliness & 36,0 & 41,1 & 48,0 & 50,0 \\
\hline Hostility & 8,0 & 16,0 & 3,3 & $2,2 * *$ \\
\hline Decisiveness, will power & 74,0 & 73,3 & 54,0 & 51,1 \\
\hline Lack of will power & 0 & 0 & 2,0 & 2,2 \\
\hline Emotional stability & 38,0 & 31,1 & 18,0 & 25,6 \\
\hline Emotionality & 2,0 & 1,1 & 16,0 & 8,9 \\
\hline Refinement and culture & 74,0 & $53,3^{* *}$ & 38,0 & 38,9 \\
\hline Indiscretion & 0 & 0 & 4,0 & $0^{* *}$ \\
\hline Ability to understand and predict others & 18,0 & 21,1 & 24,0 & 22,2 \\
\hline Inability to understand and predict others & 0 & $2,2 *$ & 0 & 0 \\
\hline Simplicity & 0 & 1,1 & 0 & 0 \\
\hline Ability to use other people & 6,0 & $15,6^{*}$ & 40,0 & 41,1 \\
\hline Logic, common sense & 58,0 & $38,9 *$ & 34,0 & $18,9^{*}$ \\
\hline Recklessness & 0 & $2,2 *$ & 6,0 & 4,4 \\
\hline Intuition & 2,0 & 0 & 18,0 & 11,1 \\
\hline Quickness & 16,0 & 15,6 & 4,0 & 10,0 \\
\hline Slowness & 0 & $3,3 *$ & 0 & 0 \\
\hline Using knowledge in practice & 54,0 & 48,9 & 38,0 & $53,3 *$ \\
\hline Knowledge of life & 58,0 & $41,1^{*}$ & 32,0 & 40,0 \\
\hline Stupidity & 0 & 0 & 4,0 & 3,3 \\
\hline Clarity and fluency of speech & 10,0 & 12,2 & 6,0 & 5,6 \\
\hline Professional success & 22,0 & 30,0 & 12,0 & 21,1 \\
\hline Aptitude for leadership & 10,0 & $2,2^{*}$ & 4,0 & 1,1 \\
\hline Appropriate self-assessment & 6,0 & 4,4 & 4,0 & 2,2 \\
\hline Inappropriate self-assessment & 0 & $2,2 *$ & 0 & 0 \\
\hline Sense of humor & 22,0 & 26,7 & 10,0 & 17,8 \\
\hline Absence of sense of humor & 0 & 1,1 & 0 & 0 \\
\hline Creativity & 16,0 & $2,2 * *$ & 6,0 & 7,8 \\
\hline Attractive appearance & 6,0 & 7,8 & 44,0 & $30,0^{*}$ \\
\hline Health & 2,0 & 4,4 & 2,0 & 1,1 \\
\hline * - differences between male and fema & ples are si & $\begin{array}{l}\text { th at the level } \\
0,01(p<0.01)\end{array}$ & $5(\mathrm{p}<0.05)$ & differences are \\
\hline
\end{tabular}


Our data revealed that the structure of the preconceptions of Male Intelligence is rather unified and stable (see Figure $1)$.
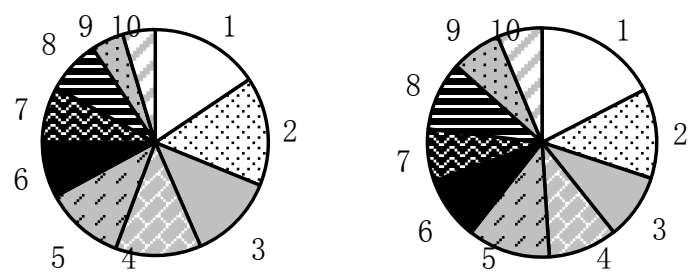

1 - Decisiveness, will power; 2 - Refinement and culture; 3 - Logic, common sense; 4 - Knowledge of life; 5 - Using knowledge in practice; 6 - Extraversion; 7 - Emotional stability; 8 - Friendliness; 9 - Professional success; 10 - Sense of humor

Figure 1. Perceived structure of Male Intelligence.

The structure of the preconceptions of Female Intelligence varies significantly from male to female respondents (see Figure 2).
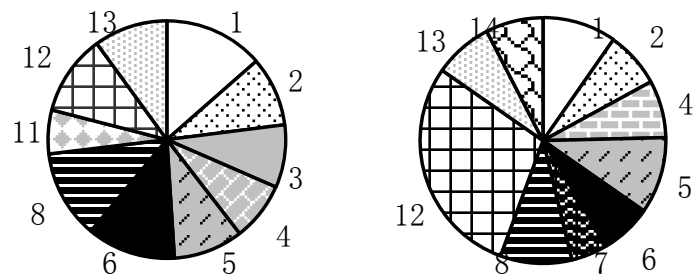

1 - Decisiveness, will power; 2 - Refinement and culture; 3 - Logic, common sense; 4 - Knowledge of life; 5 - Using knowledge in practice; 6 - Extraversion; 7 - Emotional stability; 8 - Friendliness; 11 - Ability to understand others; 12 - Attractive appearance; 13 - Ability to use other people; 14 Creativity

Figure 2. Perceived structure of Female Intelligence.

Basing on the data of content-analysis we explicated a complex of characteristics immanently attributed to both Male and Female Intelligence. This complex of features is attributed to intelligence regardless of gender. It includes personality features (Decisiveness, will power; Refinement, Friendliness, etc.) alongside with cognitive abilities (Using knowledge in practice) which are attributed to both Male and Female Intelligence (see Figure 3).

The structure of the preconceptions of Male Intelligence is rather unified and stable. It includes Logic, common sense; Emotional stability; Professional success and Sense of humor.

The Female Intelligence preconceptions have some similarity for male and female respondents but at the same time some diversity associated with gender is present. There are some common features such as Attractive appearance and Ability to use other people which were attributed to Female Intelligence by both female and male respondents. To these male respondents add Logic, common sense and Ability to understand and predict other people. In contrast female respondents add Creativity and Emotional stability to the above mentioned common qualities of Female Intelligence. Lack of uniformity in the image of Female Intelligence may be regarded to prove that intelligence is generally allocated to the sphere of masculine qualities. We suppose that diver- tive character of the data also testifies of dynamic processes in contemporary society that are accompanying formation of new gender stereotypes, including those in the sphere of implicit perceptions of Female Intelligence.

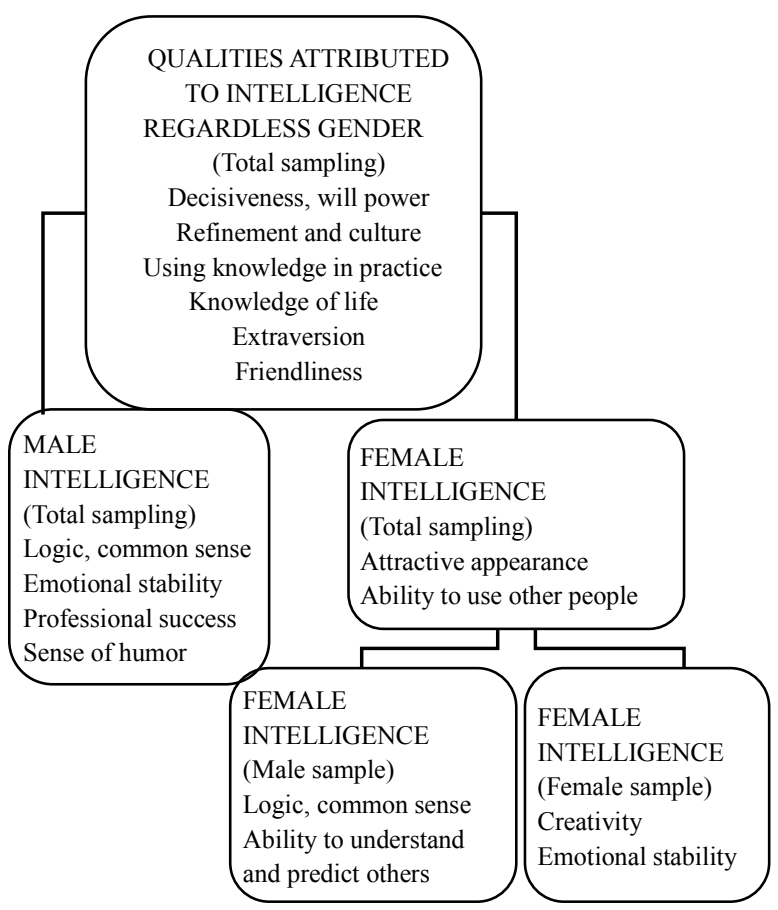

Figure 3. Implicit preconceptions of Intelligence.

Our next step was cluster analysis of the "free responces" (see Figures 4- 7 which present the results).

The hierarchical tree which shows the structure of Female Intelligence was calculated on the basis of female sample responses and it allows us to point out the next (see Figure 4).

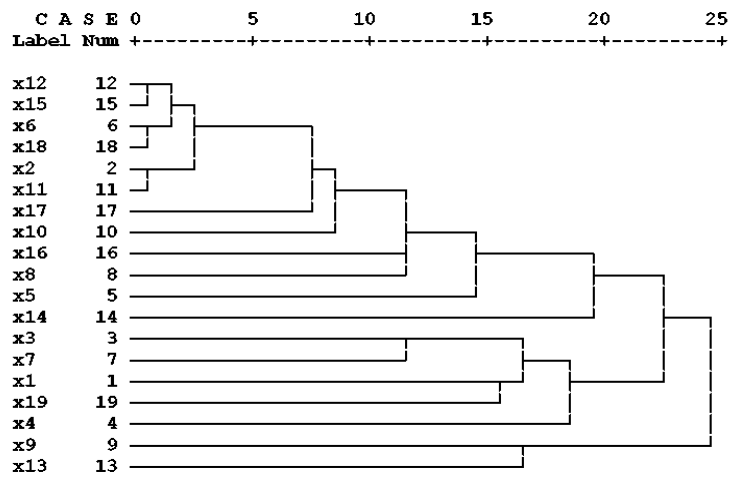

12 - Quickness; 15 - Clarity and fluency of speech; 6 - Emotionality; 18 Creativity; 2 - Unsociability; 11 - Intuition; 17 - Sense of humor; 10 Logic, common sense; 16 - Professional success; 8 - Ability to understand and predict others; 5 - Emotional stability; 14 - Knowledge of life; 3 Friendliness; 7 - Refinement and culture; 1 - Extraversion; 19 - Attractive appearance; 4 - Decisiveness, will power; 9 - Ability to use other people; 13 - Using knowledge in practice

Figure 4. Structure of Female Intelligence (female sample).

First of all the close connections should be noted between the categories in pairs:

Quickness - Clarity and fluency of speech;

Emotionality - Creativity; 
Unsociability - Intuition.

The resulting pairs in a due course are also closely connected so that all six categories are united, forming the core of the representation of Female Intelligence, as it is perceived by female respondents: Lively, receptive, creative and deep. With this core a number of qualities are associated: Sense of humor; Logic, common sense; Professional success; Ability to understand and predict other people; Emotional stability; Knowledge. This complex of qualities we labeled Complex № 1 .

Complex № 2 is constituted by a number of not too closely connected categories but apart from Complex № 1 and from other categories: Friendliness; Refinement and culture; Extraversion; Attractive appearance; Decisiveness, will power. These features are applied to the outward appearance of a clever woman, assessing her behavior and interpersonal relationships.

Complex № 3 is constituted by two categories which are applied to the active efficiency: Ability to use other people and Using knowledge in practice.

Further on we will consider the structure of Female Intelligence as it assessed by male respondents (see Figure 5).

The core of representation is formed by two closely connected pairs of categories:

Emotional stability - Sense of humor;

Hostility - Professional success, which are associated with Intuition.

This "careerist" core is accompanied by a number of qualities: Emotionality; Logic, common sense; Knowledge.

Resulting Complex № 1 is to some extent alike Complex № 1 in the described above structure of Female Intelligence as assessed by women, but the differences are substantial, and these are less differences in the elements involved than in the structure, in the type of connections between the elements, their relation to the core or to the peripheral zones.

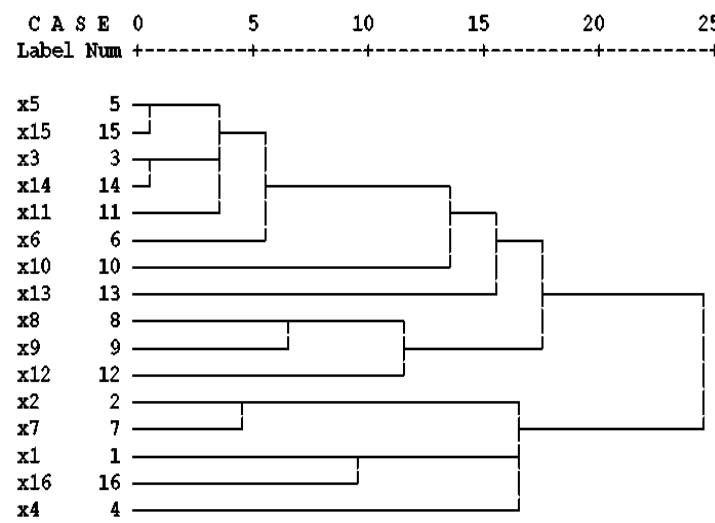

5 - Emotional stability; 15 - Sense of humor; 3 - Hostility; 14 - Professional success; 11 - Intuition; 6 - Emotionality; 10 - Logic, common sense; 13 - Knowledge of life; 8 - Ability to understand and predict others; 9 Ability to use other people; 12 - Using knowledge in practice; 2 - Friendliness; 7 - Refinement and culture; 1 - Extraversion; 16 - Attractive appearance; 4 - Decisiveness, will power

Figure 5. Structure of Female Intelligence (male sample).

Connected categories: Ability to understand and predict other people; Ability to use other people; Using knowledge in practice - form Complex № 3, which is much the same as ascribed to women's assessment of Female Intelligence. But assessed by male subjects Complex № 1 and Complex № 3 are more closely connected.

Complex № 2 is virtually the same for male and female respondents: Friendliness; Refinement and culture; Extraversion; Attractive appearance; Decisiveness, will power. However in the men's opinion this complex is separated from other characteristics to a greater degree.

Thus it can be seen that for male and female samples representations of Female Intelligence in some aspects are definitely similar and at the same time there are significant differences. In both cases the representation is constituted by three complexes of qualities two of which are similar (Complexes № 2 and 3). As for the complex № 1, its structure varies. Hostility closely connected with Professional Success takes the central place in male representation of Female Intelligence assessed by male respondents. In the representation assessed by women animosities are not presented, and the Professional success is substantially further from the core.

Thus it is possible to assert that from the male point of view Female Intelligence has some negative tint unlike its representation by female respondents.

Unlike it was in the structure of representation of Female Intelligence, where we had noted presence of three complexes of qualities, only two complexes are presented in the structure of Male Intelligence as assessed by male respondents (see Figure 6).

The core of the first is constituted by a closely connected pair of categories: Aptitude for leadership - Creativity. This pair is adjoined closely by the characteristic Quickness together with the pair of categories closely enough connected to one another: Ability to understand and predict other people - Clarity and fluency of speech. The named properties form a core which "accompanying" properties adjoin: Humor; Professional success; Extraversion; Friendliness; Emotional stability. Thus the image of a creative leader appears. We name the association of qualities Complex № 1 .

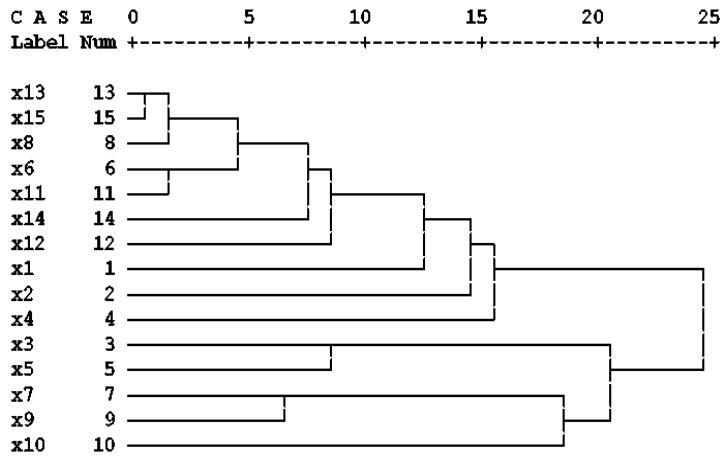

13 - Aptitude for leadership; 15 - Creativity; 8 - Quickness; 6-Ability to understand and predict others; 11 - Clarity and fluency of speech; 14 Sense of humor; 12 - Professional success; 1 - Extraversion; 2 - Friendliness; 4 - Emotional stability; 3 -Decisiveness, will power; 5 - Refinement and culture; 7 - Logic, common sense; 9 - Using knowledge in practice; 10 - Knowledge of life

Figure 6. Structure of Male Intelligence (male sample). 
The second complex of qualities includes: Decisiveness, will power; Refinement and culture; Logic, common sense; Using knowledge in practice, which is connected with the category of Knowledge. Given Complex № 2 is similar to the complex with the same name explicated in the analysis of the representation of Female Intelligence.

Then we considered the structure of Male Intelligence as assessed by female respondents (see Figure 7).

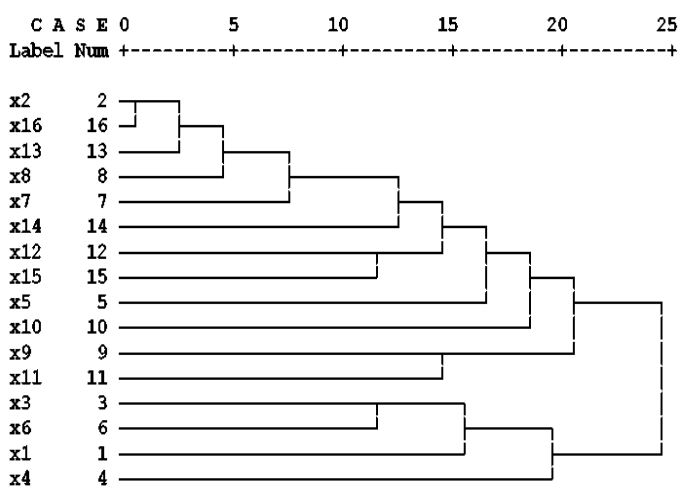

2 - Unsociability; 16 - Attractive appearance; 13 - Clarity and fluency of speech; 8 - Ability to use other people; 7 - Ability to understand and predict others; 14 - Professional success; 12 - Knowledge of life; 15 - sense of humor; 5 - Emotional stability; 10 - Quickness; 9 - Logic, common sense; 11 - Using knowledge in practice; 3 - Friendliness; 6 - Refinement and culture; 1 - Extraversion; 4 -Decisiveness, will power

Figure 7. Structure of Male Intelligence (female sample).

Here we see a two-component structure again. Complex of categories which we named "Complex № 2" composed of: Friendliness; Refinement and culture; Extraversion; Decisiveness, will power. It can be noted that we saw a similar complex in all cases of our analysis.

The core of complex № 1 is constituted by a closely connected pair: Unsociability; Attractive appearance. The categories Clarity and fluency of speech and Ability to use other people are connected with this pair. This core structure acquires some peripheral elements: Ability to understand and predict other people; Professional success; Knowledge; Sense of humor; Emotional stability; Quickness; Logic, common sense; Using knowledge in practice.

The analysis of the four hierarchical trees described above (Male Intelligence assessed by male respondents; Female Intelligence assessed by male respondents; Male Intelligence assessed by female respondents; Female Intelligence assessed by female respondents) has shown that in general three-component structure of the representation of Female Intelligence differs from two-component structure of the representation of Male Intelligence (for male as well as for female respondents).

Representation of Female Intelligence assessed both by men and by women, has a similar structure of three components two of which appear similar, while the rest, which is the basic one, differs significantly.

The results revealed some constant associations of characteristics in the implicit preconceptions of intelligence.

First of all complex which structure in all cases appears similar was allocated. This complex was named by us Complex № 2: Friendliness; Refinement and culture; Extraversion; Decisiveness, will power. As such this complex is presented in three of the four hierarchical trees, with an exception of a hierarchical tree reflecting the image of Male Intelligence as assessed by male respondents where the given complex is reduced to Refinement and culture; Decisiveness, will power. In the representation of Female Intelligence the given complex both for male and female respondents includes also the quality Attractive appearance closely connected with Extraversion. A close connection between Refinement and culture and Decisiveness, will power reserves special attention: Here we can note a withdrawal from the image of an intellectual as a weak-willed and incapable of active actions person rather popular in Russian culture of the past decades. Attractive appearance in the representation of Male Intelligence is closely connected as assessed by the female part of sample with Unsociability and forms the core of the Complex № 1.

It should be noted that Creativity was attributed by our subjects only to one's own gender! It has been revealed in the representation of Female Intelligence assessed by female respondents and in the representation of Male Intelligence, assessed by male respondents. In both cases Creativity is a part of a similar complex of qualities. For men it is: Creativity - Aptitude for leadership - Quickness - Ability to understand and predict other people - Clarity and fluency of speech.

Creativity as part of the representation of Female Intelligence is associated with the following qualities: Creativity Emotionality - Quickness - Clarity and fluency of speech Unsociability - Intuition and also is a part of Complex № 1.

In the representation of Female Intelligence assessed by male respondents a special characteristic is present - Hostility, which is closely connected with Professional success and with Emotional stability and humor, thus, forming the core of a Complex № 1.

Intuition and Emotionality which are not present in the representation of Male Intelligence are inherent to the representation of Female Intelligence. Here they are included into the core of a Complex № 1.

\section{Experiment 2}

In the second experiment we use rating procedure which was based on the results of the first experiment.

\subsection{Method}

We chose two sets of 7-point rating scales. First set for 21 personality features, well adapted to Russian sample and widely used under the name "Personality Semantic Differential" (PSD) [14]. The second one was specially constructed for evaluating cognitive abilities. It includes the following 7-points scales: Using knowledge in practice; Clarity and fluency of speech; Ability to coordinate various points of 
view; Quest for information; Knowledge of life; Quick wits, abstract thinking; Ability to understand and predict others; Cunning; Ability to use other people.

To shed some new light upon the question of personality features associated with Male and Female Intelligence our subjects were asked to evaluate personality features attributed to Clever Man and Clever Woman using the PSD scales.

Then they were asked to evaluate intellectual abilities of Clever Man (CM), Clever Woman (CW), Ordinary Man (OM) and Ordinary Woman (OW) using the PSD set of rating scales. We considered necessary to use these four personages for investigating lay conceptions of Male and Female intellect because we supposed that there might be differences in commonsense theories concerning the distribution of cognitive abilities between the male and female samples. So that Clever and Ordinary persons could be more or less different one from another. That might give us some additional information.

\subsection{Data Analysis}

The data obtained were processed by methods of descriptive statistics, analysis of the statistical significance of differences, and methods of correlation and factor analysis.

\subsection{Results and Discussion}

Analysis of the results of evaluating of Male and Female
Intelligence as ascribed to Clever Man and Clever Woman for the total sampling revealed statistically significant differences in concern of some cognitive abilities in the structure of Male and Female Intelligence (see Table 2).

Male Intelligence is credited with a greater capacity of Using knowledge in practice. Female is superior about the last three parameters listed in the inventory, which can be attributed to the sphere of social intelligence.

Implicit representations of Male and Female Intelligence differ substantially for male and female samples. It is noteworthy that there is no cognitive ability in which men would recognize the superiority of women! Men take credit for excellence in Using knowledge in practice, Ability to coordinate various points of view, Knowledge of life and Quick wits, abstract thinking. They also deny the superiority of women in relation to the "social" skills. Women recognize the priority of men in the Using knowledge in practice, but consider the difference less pronounced than the total sampling. In addition, women tend to see female's superiority in regard to "social" parameters as more substantial in comparison with the data for the total sampling. It should be noted that Quick wits, abstract thinking women suppose to be significantly more manifested in Female Intelligence, while the male sample regards Male Intelligence superior in this respect.

Table 2. Estimates of Male and Female Intelligence Ascribed to Clever Man (CM) and Clever Woman (CW).

\begin{tabular}{|c|c|c|c|c|c|c|}
\hline \multirow{2}{*}{ Characteristic } & \multicolumn{2}{|c|}{ Total Sampling } & \multicolumn{2}{|c|}{ Male Sample } & \multicolumn{2}{|c|}{ Female Sample } \\
\hline & $\mathrm{CM}$ & $\mathrm{CW}$ & $\mathrm{CM}$ & $\mathrm{CW}$ & $\mathrm{CM}$ & $\mathrm{CW}$ \\
\hline Using knowledge in practice & 6,61 & $6,38^{*}$ & 6,52 & $5,78^{*}$ & 6,63 & $6,50 * * *$ \\
\hline Clarity and fluency of speech & 6,30 & 6,34 & 6,20 & 6,10 & 6,32 & 6,40 \\
\hline Ability to coordinate various points of view & 6,43 & 6,41 & 6,44 & $6,12 * * *$ & 6,43 & 6,47 \\
\hline Quest for information & 6,60 & 6,52 & 6,46 & 6,34 & 6,63 & 6,55 \\
\hline Knowledge of life & 6,50 & 6,40 & 6,50 & $5,94 * *$ & 6,50 & 6,50 \\
\hline Quick wits, abstract thinking & 6,40 & 6,39 & 6,56 & $5,78^{*}$ & 6,37 & $6,52 * * *$ \\
\hline Ability to understand and predict others & 6,13 & $6,43 *$ & 6,14 & 5,80 & 6,13 & $6,57 *$ \\
\hline Cunning & 5,20 & $6,07 *$ & 5,60 & 5,86 & 5,12 & $6,11^{*}$ \\
\hline Ability to use other people & 5,24 & $5,72 *$ & 5,64 & 5,76 & 5,16 & $5,71 *$ \\
\hline
\end{tabular}

Table 3. Estimates of Male and Female Intelligence Ascribed to Ordinary Man (OM) and Ordinary Woman (OW).

\begin{tabular}{|c|c|c|c|c|c|c|}
\hline \multirow{2}{*}{ Characteristic } & \multicolumn{2}{|c|}{ Total Sampling } & \multicolumn{2}{|c|}{ Male Sample } & \multicolumn{2}{|c|}{ Female Sample } \\
\hline & OM & OW & $\mathrm{OM}$ & OW & $\mathrm{OM}$ & OW \\
\hline Using knowledge in practice & 4,88 & 4,80 & 5,16 & $4,08^{*}$ & 4,82 & 4,95 \\
\hline Clarity and fluency of speech & 4,73 & $4,99 * *$ & 5,08 & 4,88 & 4,66 & $5,02 *$ \\
\hline Ability to coordinate various points of view & 4,66 & 4,76 & 4,86 & $4,16^{*}$ & 4,61 & $4,89 * * *$ \\
\hline Quest for information & 4,72 & 4,84 & 5,08 & $4,38 * *$ & 4,65 & $4,94 * *$ \\
\hline Knowledge of life & 4,80 & 4,87 & 5,36 & $4,56^{*}$ & 4,68 & $4,94 * *$ \\
\hline Quick wits, abstract thinking & 4,46 & $4,83 *$ & 4,88 & $4,24 * * *$ & 4,37 & $4,96 *$ \\
\hline Ability to understand and predict others & 4,32 & $5,14^{*}$ & 4,64 & 4,76 & 4,25 & $5,23 *$ \\
\hline Cunning & 4,33 & $5,47 *$ & 4,56 & $5,26 * * *$ & 4,28 & $5,51 *$ \\
\hline Ability to use other people & 4,36 & $4,99 *$ & 4,28 & $5,02 * *$ & 4,38 & $4,99 *$ \\
\hline $\begin{array}{l}* \text { - differences between estimates for OM } \\
\text { ences are significant at the level of } 0,01(\mathrm{p}\end{array}$ & & 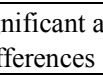 & leve & 0,001 & 01); & $\begin{array}{l}\text { differ- } \\
(p<0,05)\end{array}$ \\
\hline
\end{tabular}


Table 4. Personality Qualities of Clever Man (CM) and Clever Woman (CW) (Factors of Semantic Differential Scale).

\begin{tabular}{|c|c|c|c|c|c|c|}
\hline \multirow{2}{*}{ Factor } & \multicolumn{2}{|c|}{ Total Sampling } & \multicolumn{2}{c|}{ Male Sample } & Female Sample \\
\cline { 2 - 7 } & $\mathrm{CM}$ & $\mathrm{CW}$ & $\mathrm{CM}$ & $\mathrm{CW}$ & $\mathrm{CM}$ & $\mathrm{CW}$ \\
\hline 1. Evaluation & 40,57 & $41,55^{* *}$ & 39,98 & 39,92 & 40,69 & $41,88^{*}$ \\
\hline 2. Potency & 39,04 & $36,81^{*}$ & 39,26 & $33,34^{*}$ & 39 & $37,53^{*}$ \\
\hline 3. Activity & 33,5 & 34,3 & 32,48 & 33,41 & 33,72 & 34,52 \\
\hline * - differences between estimates for CM and CW are significant at the level of \\
0,001 (p $\leq 0,001) ; * *$ - differences are significant at the level of 0,01 (p<0,01) \\
\hline
\end{tabular}

Table 5. Personality Qualities of Clever Man (CM) and Clever Woman (CW) (Scales of Semantic Differential).

\begin{tabular}{|c|c|c|c|c|c|c|}
\hline \multirow{2}{*}{ Scale } & \multicolumn{2}{|c|}{ Total Sampling } & \multicolumn{2}{c|}{ Male Sample } & \multicolumn{2}{c|}{ Female Sample } \\
\cline { 2 - 8 } & CM & CW & CM & CW & CM & CW \\
\hline Unattractive - Attractive & 5,68 & $6,26^{*}$ & 5,64 & 5,82 & 5,68 & $6,35^{*}$ \\
\hline Weak - Strong & 5,84 & $5,0^{*}$ & 5,64 & $4,44^{*}$ & 5,88 & $5,12^{*}$ \\
\hline Tacit - Talkative & 4,8 & $5,12^{*}$ & 4,28 & $4,92^{* * *}$ & 4,91 & $5,18^{* * *}$ \\
\hline Negligent - Conscientious & 6,35 & $6,13^{* *}$ & 6,32 & $5,88^{* * *}$ & 6,35 & $6,18^{* * *}$ \\
\hline Yielding - Obstinate & 4,24 & 4,13 & 4,72 & $3,68^{*}$ & 4,14 & 4,23 \\
\hline Reserved - Frank & 5,14 & $5,39^{* *}$ & 5,06 & 5,36 & 5,15 & $5,40^{* * *}$ \\
\hline Selfish -Unselfish & 5,46 & 5,47 & 5,60 & 5,14 & 5,43 & 5,53 \\
\hline Dependent - Independent & 5,85 & $5,56^{*}$ & 5,76 & $4,84^{*}$ & 5,87 & 5,71 \\
\hline Passive - Active & 6,44 & $6,1^{*}$ & 6,36 & $5,40^{*}$ & 6,46 & $6,25^{*}$ \\
\hline Callous - Responsive & 5,53 & $5,81^{* *}$ & 5,08 & $5,8^{* *}$ & 5,62 & $5,81^{* *}$ \\
\hline Hesitating - Resolute & 6,4 & $6,07^{*}$ & 6,42 & $5,36^{*}$ & 6,4 & $6,21^{* *}$ \\
\hline Sluggish - Energetic & 6,14 & 6,09 & 6,06 & $5,58^{* * *}$ & 6,16 & 6,20 \\
\hline Unfair - Fair & 6,08 & 6,07 & 6,06 & $5,54^{* * *}$ & 6,09 & 6,18 \\
\hline Relaxed - Tense & 3,91 & $3,6^{*}$ & 3,96 & 3,54 & 3,90 & $3,61^{*}$ \\
\hline Quiet - Fussy & 2,53 & $2,78^{* * *}$ & 2,18 & $2,84^{* *}$ & 2,60 & 2,77 \\
\hline Unfriendly - Friendly & 5,62 & $5,99^{*}$ & 5,54 & 5,88 & 5,64 & $6,01^{*}$ \\
\hline Uncertain - Confident & 6,41 & 6,29 & 6,34 & $5,78^{* * *}$ & 6,43 & 6,40 \\
\hline Unsociable - Sociable & 5,81 & $6,02^{* *}$ & 5,74 & 6,00 & 5,82 & $6,03^{* * *}$ \\
\hline Dishonest - Honest & 5,85 & 5,82 & 5,74 & 5,86 & 5,88 & 5,82 \\
\hline Not self-sufficient - Self-sufficient & 6,39 & $6,16^{*}$ & 6,42 & $5,70^{* *}$ & 6,38 & $6,25^{* * *}$ \\
\hline Unflappable - Irritable & 2,64 & $2,8^{* *}$ & 2,80 & $3,32^{* * *}$ & 2,61 & 2,69 \\
\hline$*$ - differences between estimates for CM and CW are significant at the level of $0,001(\mathrm{p} \leq 0,001) ;$ \\
$* *$ - differences are significant at the level of $0,01(\mathrm{p}<0,01) ; * * *-$ differences are significant at the \\
\hline
\end{tabular}

Below results of the evaluation for the Male and Female Intelligence as ascribed to Ordinary Man and Ordinary Woman are considered, as shown in Table 3. Noteworthy, in this case all significant differences for the total sampling are in favor of Female Intelligence! Male sample recognizes the superiority of Female Intelligence in respect of the three "social" parameters (significant differences only for two out of three). In respect of the other abilities men consider themselves superior. All differences are statistically significant with the exception of the Clarity and fluency of speech.

Female sample favored Female Intelligence on all parameters, except for the Using knowledge in practice, where there are no significant differences.

Our next step will be analyzing results of Personality Semantic Differential scales (see Table 4). Higher ratings of Potency factor were more stably ascribed to Male Intelligence. Female Intelligence is stably characterized by higher ratings in Evaluation. Activity factor evaluation shows no significant differences. The results of our analysis of various categories of Personality Differential allowed us to supple- ment the data existing in the literature, with the fact that in laymen's view, Female Intelligence structure includes aside from "social" abilities some expressive characteristics (responsive, talkative). Instrumental and activity characteristics mainly prevail in the perception of Male Intelligence (conscientious, strong, decisive, independent and active).

More detailed assumption of personality features ascribed to Clever Man and Clever Woman with all the personality scales shows that statistically significant differences were found across a large number of parameters (see Table 5).

The results revealed some categories consistently attributed to one of the sexes, which are confirming the literature data on the prevalence in the perception of Male Intelligence of instrumental and activity characteristics, and in representations of Female Intelligence - of expressive and communicative ones (see Table 6).

It should be noted that alongside with the absence of significant differences in the factor of "activity", its' constitutive components are different: For Female Intelligence it is Talkative, Sociable and Frank, for Male Intelligence it is Active, Quiet and Unflappable. 
Table 6. Characteristics Attributed to Male and Female Intelligence.

\begin{tabular}{|c|c|c|c|}
\hline \multirow{2}{*}{ Representation } & \multicolumn{3}{|c|}{ Factors of Semantic Differential Scale } \\
\hline & Evaluation & Potency & Activity \\
\hline Female Intelligence $(\mathrm{CW})$ & $\begin{array}{l}\text { Attractive Friendly } \\
\text { Responsive }\end{array}$ & Relaxed & $\begin{array}{c}\text { Talkative Sociable } \\
\text { Frank }\end{array}$ \\
\hline Male Intelligence (CM) & Conscientious & $\begin{array}{l}\text { Strong Resolute Independent } \\
\text { Self-sufficient }\end{array}$ & $\begin{array}{l}\text { Active Quiet } \\
\text { Unflappable }\end{array}$ \\
\hline
\end{tabular}

In order to reveal the general structure of lay representations of Male and Female Intelligence correlations were calculated between the estimates of cognitive abilities and personality features ascribed to $\mathrm{CM}$ and $\mathrm{CW}$ and factor analysis was accomplished, separately for the male sample and for the female sample answers. Factorization estimates were nine cognitive abilities for $\mathrm{CM}, \mathrm{OM}$ and $\mathrm{CW}$ and $\mathrm{OW}$, as well as indicators of the factors "Evaluation", "Potency", "Activity" from the PSD.

Below the results of Factor analysis of female sample evaluations are considered which reveal five factors.

Factor I we interpreted as "Ingenuousness, Simple-mindedness - Slyness, Cunning". It is noteworthy that at the positive pole of factor I there are all the cognitive abilities as ascribed to Ordinary Man, except for the last two abilities mentioned in the questionnaire: Cunning and the Ability to use other people. The latter "manipulative" abilities for all the four assessed images are closer to the negative pole. Such factor loadings allow us to offer the listed above interpretation of this factor.

Note that in all our calculations we revealed a stable tendency to isolation of "manipulative" skills and even their opposition of the other intellectual abilities. The most "quirky" in the views of respondents is a Clever Woman, behind her is a Clever Man. The most straightforward is Ordinary Man, and adjoining him an Ordinary Woman.

Factor II we interpreted as "Wisdom, Understanding of Life - Vanity. Feminine Aspects of Wisdom". Factor III we interpreted as "Superficiality, shallow mind - Deep mind. Masculine Aspects of Wisdom ". The difference between factor III and Factor II is that for the former "manipulative" cognitive abilities are untapped, i.e., this factor seems less "ethically loaded." The opposition of "manipulative" characteristics to other cognitive abilities is more pronounced in respect to Factor II.

In respect to Factor III Ordinary Woman (as the embodiment of superficiality) and Clever Man (as the embodiment of the depth) are opposed, while in respect to the Factor II Clever Woman (the embodiment of wisdom) is opposed to the Ordinary Man (embodying the vanity).

Our results are consistent with the data on gender and wisdom research[15,16,17]. The research of Ardelt[15] revealed that implicit preconceptions of feminine and masculine aspects of wisdom differed so that women tended to score higher on the affective dimension of wisdom than men. By contrast, men tended to outperform women on the cognitive wisdom dimension.

The research of Glück J, Strasser I. \& Bluck S.[16] showed similar gender differences in participants' reports of an event in which they were wise: men most often reported events from their professional life whereas women reported events from a range of domains including family-related events and events concerning death or illness. Aspects of concern for others were rated as slightly more typical for a female wise person, in contrast with a slightly more cognition-oriented view of wisdom in men.

Factor IV reveals a tendency for appreciation of personal qualities ascribed to clever people. In this case the positive pole includes all factors from the PSD ("Evaluation", "Potency", "Activity") for Clever Man and Clever Woman whereas almost all of the others characteristics tend to the negative pole.

It seems possible to interpret Factor $\mathrm{V}$ as Machiavellianism. Here again reveals itself a tendency to isolate "manipulative" abilities from other intellectual abilities.

Factor analysis of male sample data allow us to describe the following three factors.

Factor I is very similar to that calculated on a female sample. Here, however, factor loadings for the abilities of Clever Man and Ordinary Man show differences to a lesser extent than in the representation of female sample. Clever Man and Ordinary Man images are merged to a greater extent. To a lesser extent are differentiated images of Clever Man and Clever Woman too. The latter indicates less gender-coloring of intellectual abilities in the view of men than in the view of women.

In respect to Factor II Clever Woman is opposed to the three other images: Clever Man, Ordinary Woman and Ordinary Man. It should be noted that all the intellectual abilities of $\mathrm{CW}$, listed in the questionnaire, here lie close to each other so that even "manipulative" ability is not detached from the main group. It seems that in this case a particular gender stereotype has been revealed characteristic to the male sample: Clever Woman is perceived as an anomaly in contrast to the other images of the "normal" people. It is possible to interpret this factor as follows: "Clever Woman as Anomaly".

Factor III it is possible to interpret as Machiavellianism, similar to Factor $\mathrm{V}$ for the female sample. A tendency to isolate the "manipulative" abilities from other intellectual abilities and even to oppose them to other abilities was bare once again.

\section{Conclusions}

Our investigation revealed implicit preconceptions of Male and Female Intelligence in contemporary Russian society. A substantial difference in the structure and constituting elements between the representations was shown. It was shown also that the preconceptions of Male and Female 
Intelligence are substantially different for male and for female samples.

Using factor analysis method, separately for men's answers and women's answers, we exposed and described the structure of lay preconceptions of Male and Female Intelligence. It was shown that alongside with common factors there exist specific ones as well, that define the structure of lay preconceptions of Male and Female Intelligence for male and for female sampling. Two common factors were detected for the respondents of both genders: "Ingenuousness, Simple-mindedness - Slyness, Cunning" and "Machiavellis $\mathrm{m} "$.

When analyzing the women's answers, we detected factors not present in the male sample: "Wisdom, Understanding of Life - Vanity. Feminine Aspects of Wisdom" and "Superficiality, shallow mind - Deep mind. Masculine Aspects of Wisdom ". Analysis of male sample answers did not reveal similar factors. Instead a specific factor was exposed, which we interpreted as "Clever woman as Anomaly". The reason for that might be culture biased and perhaps interrelated with the fact that our results revealed the absence of a generally known tendency to lower self-esteem of intellectual abilities for women[4,5]. In contrast a marked tendency for female sample to evaluate Female Intelligence in all its' aspects and functions superior to the Male Intelligence was discovered. This tendency is particularly manifest in comparison of evaluations of cognitive abilities of Ordinary Woman and Ordinary Man, less striking was the difference between evaluations of abilities of Clever Man and Clever Woman.

It's noteworthy that the facts exposed are consistent with the results of the research of self-evaluations of intellectual abilities of women in Slovakia[18], where woman awarded themselves higher overall scores on intellectual abilities, in contrast to other European woman. We agree that: "...it may well be that under the pressure of socialist governments of Eastern Europe the role of females in society was somewhat different from that in capitalist Western Europe, where they took a more active role in the economy and were socialized differently in school" [18, p. 137]. We suppose also that this tendency to a large degree is due to the tradition of a very active and even leading role that women played in the family life in Russia in the second half of the 20th century[19] and also due to massive propaganda of equal rights and abilities of men and women of the Soviet period[20].

\section{REFERENCES}

[1] Furnham, A., 2000, Thinking about intelligence. The Psychologist, 13(10), 510-514

[2] Jensen, A., 1998, The $\mathrm{g}$ factor: The science of mental ability. Westport, CT: Praeger

[3] Furnham, A., Clark, K., \& Bailey, K., 1999, Sex differences in estimates of multiple intelligences. European Journal of Personality, 247-259
[4] Furnham, A., 2005, Gender and Personality Differences in Self- and Other Ratings of Business Intelligence. British Journal of Management, 16, 91-103

[5] Furnham, A., Crawshaw, J., \& Rawles, R., 2006, Sex Differences in Self-Estimates on Two Validated IQ Test Subscale Scores. Journal of Applied Social Psychology, 36: 417-440.

[6] Rammstedt, B., \& Rammsayer, T. H., 2002, Gender differences in self-estimated intelligence and their relation to gender-role orientation. European Journal of Personality, 16, 369-382

[7] Sternberg, R. J., 2003, Contemporary Theories of Intelligence. Handbook of Psychology, 21-45

[8] Boddice, R., 2011, The Manly Mind? Revisiting the Victorian "Sex in Brain" Debate. Gender \& History, 23, 321-340

[9] Dustin B. Thoman, Paul H. White, Niwako Yamawaki and Hirofumi Koishi, 2010, Variations of Gender-math Stereotype Content Affect Women's Vulnerability to Stereotype Threat. Sex Roles, Volume 58, Numbers 9-10, 702-712

[10] Azuma, H., \& Kaschiwagi, K., 1987, Descriptors for an intelligent person: A Japanese Study. Japanese Psychological Research, 29, 17-26

[11] Miguel, I., Pires Valentim, J., Carugati, F., 2010, Intelligence and its development: Social representations and social identities. Papers on Social Representations, 19, 20.1-20.33 [Online]. Available: http://www.psych.lse.ac.uk/psr

[12] Roets, A., Van Hiel, A. and Dhont, K., 2011, Is Sexism a Gender Issue? A Motivated Social Cognition Perspective on Men's and Women's Sexist Attitudes Toward Own and Other Gender. European Journal of Personality. doi:

10.1002/per.843

[13] Swami, V., Furnham, A., Maakip, I., Ahmad, M. S., Nawi, N. H. M., Voo, P. S. K., Christopher, A. N. and Garwood, J., 2008, Beliefs about the meaning and measurement of intelligence: a cross-cultural comparison of American, British and Malaysian undergraduates. Applied Cognitive Psychology, 22: $235-246$

[14] Fetiskin, N.P., Kozlov, V.V., \& Manujlov, G.M., 2005, Socio-psychological diagnostics of personality development and small groups (in Russian). Moscow, Russia: Institute of Psychotherapy

[15] Ardelt M., 2009, How Similar are Wise Men and Women? A Comparison Across Two Age Cohorts. Research in Human Development, 6 (1), Special Issue: Gender and Wisdom, 9-26

[16] Glück J, Strasser I. \& Bluck S., 2009, Gender Differences in Implicit Theories of Wisdom. Research in Human Development, 6 (1), Special Issue: Gender and Wisdom, 27-44

[17] Levenson, M., 2009, Gender and Wisdom: The Roles of Compassion and Moral Development. Research in Human Development, 6 (1), Special Issue: Gender and Wisdom, 45-59

[18] Furnham, A., Rakow, T., Sarmany-Schiller, \& De Fruyt, F., 1999, European differences in self-perceived multiple intelligences. European Psychologist, 4, 131-138

[19] Druzhinin, V.N., 2005, Psychology of Family Relations (in Russian). St.Petersburg, Russia: Piter

[20] Kletzyna, I.S., 2004, Psychology of Gender Relations (in Russian). Rusia, St.Petersburg: Aleteja 